\section{Sublingual hoch dosieren!}

$\mathrm{N}$ och ist die Datenlage für die sublinguale Immuntherapie (SLIT) weniger gut als die für die subkutane Immuntherapie (SCIT). So habe man erst im Laufe der Jahre erkannt, dass eine hohe Dosierung wesentlich sei, berichtete Prof. Dr. Gerhard Schultze-Werninghaus, Bochum. Jetzt sei zumindest die durch Gräserpollen ausgelöste Rhinokonjunktivitis eine Indikation für die SLIT, für andere Allergene sowie für den Einsatz bei Asthma oder im Kindesalter forderte er weitere Studien.

Zur SLIT bei Gräserpollenallergie stellte Priv.-Doz. Dr. Jörg Kleine-Tebbe, Berlin, eine aktuelle multizentrische randomisierte Doppelblindstudie mit dem hochdosierten Gräserextrakt AllerSlit ${ }^{\circledR}$ forte vor. In der Pollensaison 2003 wurden die Beschwerden der Teilnehmer als Basiswert erfasst, die Behandlungsphase erstreckte sich dann über die Jahre 2004 und 2005. Insgesamt 89 Patienten, 41 im aktiven und 48 im Plazeboarm, wa- ren am Studienende auswertbar. In der Saison 2003 war der kombinierte Symptom-Medikations-Score in beiden Studienarmen vergleichbar gewesen. Nach 18 Behandlungsmonaten, in der Pollensaison 2005, hatte er sich in der Plazebogruppe nicht signifikant verändert, dagegen hatte er unter Verum hochsignifikant abgenommen $(\mathrm{p}=0,0005)$. Bezog man in einer Intention-to-treat-Analyse alle Patienten in die Auswertung mit ein, also auch die Studienabbrecher, blieb das Ergebnis weiter signifikant, betonte Kleine-Tebbe. Zudem wurde auch eine etwa zehnfache Erhöhung des IgG Levels in der Verumgruppe festgestellt, was eine entsprechende Reaktion des Immunsystems belegt.

$f k$

Symposium „Allergologie und SIT: Fakten, Tendenzen und Strategien", unterstützt von Allergopharma, Reinbek. Zweiter Gemeinsamer Deutscher Allergiekongress, Lübeck, 26.-29. September 2007

\title{
Schnelle Wirkung dank Clustertherapie
}

W ährend die konventionelle Aufdosierung bei der subkutanen Immuntherapie (SCIT) drei bis vier Monate in Anspruch nimmt, gelingt dies mit der Clustertherapie in wenigen Wochen. Sie kann somit noch kurz vor der Pollensaison begonnen werden. Gerade mit Allergoiden ist eine Clustertherapie gut verträglich und wirksam, stellte Dr. Enrique Fernandéz-Caldas, Madrid, fest. Er berichtete von einer randomisierten kontrollierten Studie, in der die klinische Wirksamkeit des Gräserpollenallergoids Clustoid ${ }^{\circledR}$ prospektiv drei Monate nach Therapiebeginn an Probanden mit allergischer Rhinitis aufgrund einer Sensibilisierung gegen Gräserpollen überprüft wurde: 22 Patienten erhielten eine Cluster-SCIT, elf dienten als Kontrollgruppe und erhielten keine Immuntherapie. Die Aufdosierung sah am ersten Tag sowie nach einer Woche jeweils zwei Injektionen im Abstand von 30 Minuten vor. Nach 15 Tagen und danach monatlich erhielten die Patienten dann die Erhaltungsdosis. Als objektiver Parameter der Wirksamkeit diente der nasale Provokationstest. Tatsächlich wurde in der Gruppe mit Cluster-SCIT nach drei Monaten im Mittel eine etwa doppelt so hohe Allergendosis toleriert wie zu Beginn der Studie, während dieser Wert in der Kontrollgruppe etwa gleich geblieben war. In der Kontrollgruppe war bei einem Drittel der Patienten eine Verschlechterung in Bezug auf die tolerierte Allergendosis festzustellen, nach der Cluster-SCIT trat dies in keinem Fall auf. Die Studie konnte somit belegen, dass die Cluster-Immuntherapie mit dem Gräserpollenallergoid Clustoid ${ }^{\circledR}$ bereits nach drei Monaten klinisch relevante Wirkung zeigt. $f k$

Satellitensymposium „Allergologie 2007“. Zweiter Gemeinsamer Deutscher Allergiekongress, Lübeck, 26.-29. September 2007. Veranstalter: Dr. Beckmann Pharma, Seefeld, und Roxall, Hamburg

\section{Co-Marketing für Kombinationspräparat}

Mit Wirkung zum 1. September 2007 hat der Neusser Pharmakonzern Janssen-Cilag die Rechte am Asthmapräparat Inuvair ${ }^{\circledR}$ vom Unternehmen UCB übernommen. Bei Inuvair ${ }^{\circledR}$ handelt es sich um eine fixe Kombination der Wirkstoffe Formoterol und Beclometasondipropionat, die unter dem Namen Foster ${ }^{\circledR}$ auch von Asche Chiesi vertrieben wird. Beide Unternehmen zeichnen in Zukunft gemeinsam für die Vermarktung verantwortlich.

Bei Foster ${ }^{\circledR}$ und Inuvair ${ }^{\circledR}$ liegen die Wirkstoffe nicht als Suspension, sondern in Lösung vor und können durch die extrafeine Verneblung mittels Modulite ${ }^{{ }_{-}}$ Technologie gut bis in die Lungenperipherie vordringen. Dadurch kann die Glukokortikoiddosis im Gegensatz zu früheren FCKW-Dosieraerosolen, die Beclometason enthielten, um etwa $60 \%$ reduziert werden.

Nach Informationen von Janssen-Cilag, Neuss

\section{Neue Gräser-/Roggen- Mischung}

Wenn allergische Patienten im Hauttest deutlich positiv auf Gräser und Roggen reagieren oder entsprechend erhöhte spezifische IgE-Spiegel aufweisen, kann sich eine Hyposensibilisierung mit einer Gräser-/Roggen-Mischung lohnen, denn mit ihr lässt sich ein breiteres Allergenspektrum abdecken. Zu diesem Zweck bietet das Unternehmen Allergopharma seit August das Sublingualpräparat AllerSlit ${ }^{\circledR}$ forte auch in einer Gräser-/Roggen-Mischung an, die auf einen Hauptallergengehalt von 40 pg Gruppe-5-Allergen standardisiert ist.

Die Ergebnisse der hochdosierten sublingualen Immuntherapie mit AllerSlit ${ }^{\circledR}$ forte bei Gräserpollenallergie können sich sehen lassen: Nach 18 Monaten Therapie reduzierte sich im Vergleich zu Plazebo der Symptom-Medikations-Score um $46 \%$. Im selben Zeitraum zeigte sich unter Verum eine progressive Zunahme des $\operatorname{lgG}_{4}$ Spiegels von $38 \mu \mathrm{g} / \mathrm{l}$ auf $982 \mu \mathrm{g} / \mathrm{l}$.

Nach Informationen von Allergopharma, Reinbek 